\title{
Microstructural development, electrical properties and oxygen permeation of zirconia-palladium composites
}

\author{
C.S. Chen, B.A. Boukamp, H.J.M. Bouwmeester, G.Z. Cao, II. Kruidhof, \\ A.J.A. Winnubst, A.J. Burggraaf \\ Laboratory of Inorganic Chemistry, Catalysis and Inorganic Materials Science, Faculty of Chemical Technology, University of Twente, \\ P.O. Box 217, 7500 AE, Enschede, The Netherlands
}

Received 1 September 1993; accepted for publication 7 November 1994

\begin{abstract}
Yttria-stabilized cubic zirconia (YSZ)-palladium dual phase composites have been investigated. The percolative composite containing $40 \mathrm{vol} \% \mathrm{Pd}$ (ZYPd40) showed a much larger oxygen permeability than that of the non-percolative composite containing $30 \mathrm{vol} \%$ Pd (ZYPd30). For a $2.0 \mathrm{~mm}$ thick percolative composite, an oxygen flux of $4.3 \times 10^{-8} \mathrm{~mol} / \mathrm{cm}^{2} / \mathrm{s}$ was measured at $1100^{\circ} \mathrm{C}$ with oxygen partial pressures at the feed and permeate sides being 0.209 and $0.014 \mathrm{~atm}$, respectively. This value is two orders of magnitude larger than that observed for a $2.0 \mathrm{~mm}$ thick non-percolative composite at the same temperature with the oxygen partial pressures at the feed and permeate sides being 0.209 and $1.5 \times 10^{-4} \mathrm{~atm}$, respectively. From the dependence of the oxygen permeation on the temperature and on the oxygen partial pressures, it was concluded that the transport of the oxygen ions through the YSZ phase in the percolative system was the rate limiting step.
\end{abstract}

Keywords: Oxygen permeability; Percolation; Zirconia-palladium

\section{Introduction}

There have been increasing research and development activities in the field of inorganic membrane in the past decade [1]. Inorganic membranes can be used for separation of mixtures and manipulation of chemical reactions. Dense inorganic membranes are particularly uscful becausc of their high perm-selectivities, i.e., only certain species such as hydrogen and oxygen are allowed to diffuse through the membrane. In chemical reactions this can cause considerable effects on yield and selectivity $[2,3]$.

An important dense inorganic membrane is formed

\footnotetext{
* Presented at the 9th Solid State Ionics International Conference, 12-17 September, 1993, The Hague, The Netherlands.
}

by ceramic solid electrolytes. The mobile species are cations or anions (oxygen, hydrogen). The flux of ions is driven either by an electric field or by a pressure gradient. In the first case the material is preferably electronically insulating (ionic transference number $t_{\mathrm{ion}}=1$ ) [4]. If the reaction is pressure driven (no external electric field), mass transport (permeability) is only possible if ions (or ion vacancies) and electrons (or electron holes) move together through the solid. This implies that the material must be a mixed (electronic and ionic) conductor. The total flux of, e.g., oxygen species, is then determined by (i) the bulk permeability and (ii) the reaction rate of the species at the surface [5].

The surface reaction may consist of many sequential steps each of which may be rate limiting $[6,7]$. 
Important steps are adsorption from the gas phase, the actual charge transfer reaction between the adsorbed species and the bulk together with their reversed reactions. Upon decreasing the membrane thickness the surface reaction inevitably becomes rate limiting. Mixed conduction probably plays an important role in determining the surface reaction rate.

In order to have a good oxygen semi-permeability the material must have large ionic conductivity as well as electronic conductivity in the appropriate range of temperature and oxygen partial pressure. Moreover, the surface activity for transport of gaseous oxygen from the gas phase to the solid material should be large, i.e., the material should be a good electrode material too. It has been found difficult to realize all these requirements in one single component material. In a composite material different functions may be provided by different phases. The composite consists of two phases: one phase being a good oxygen ion conductor, the other phase being a good electronic conductor. If both phases form an infinitely continuous network, then oxygen ions can transport through the ionic conductive phase, the electrical charge being balanced by the diffusion of electronic charge carriers through the electronic conductive phase. The electronic conductive phase can also promote surface reactions.

The composite of YSZ and palladium has been chosen as a model system to examine this concept following preliminary results reported by Mazanec et al. [3]. The cubic zirconia and palladium is a good oxygen ion and electron conductor respectively. Moreover, palladium as a good catalytic metal can enhance the surface reaction. Therefore, the composite is expected to be a good material for oxygen permeation.

From the point of view of basic research as well as practical application, it is important that the material should be chemically stable and mechanically strong, and it should be relatively easy to prepare a well defined microstructure. It can be expected that YSZ-Pd composites meet these requirements. Both YSZ and Pd are chemically stable at elevated temperatures. YSZ has a relatively high strength. On the other hand, its thermal shock resistance is poor due to its low thermal conductivity. Palladium has a relatively low strength, but it has a good thermal shock resistance. Probably, the ceramic and metal compo- nent in the composite can benefit from each other in these aspects.

This paper is to briefly describe the preparation, microstructural development, electrical properties and oxygen permeability of the relatively coarse grained YSZ-Pd composite. The fine grained materials are under investigation and the results will be compared with this work in a subsequent paper.

\section{Experimental}

The mixtures of YSZ (TZ8Y, Tosoh) and PdO powders (Cerac Inc.) were milled in acetone using zirconia balls. The $\mathrm{PdO}$ in the mixtures were reduced to $\mathrm{Pd}$ under a flow of hydrogen/nitrogen at $600^{\circ} \mathrm{C}$. The as-obtained powders were isostatically pressed at $400 \mathrm{MPa}$ and sintered at temperatures between 1350 $1480^{\circ} \mathrm{C}$ in the furnace of a dilatometer (NETSCH402E) under flowing nitrogen. For comparison, an YSZ ceramic was prepared under similar conditions. The densities of the composites were determined by the Archimedes technique (in $\mathrm{Hg}$ ). The sintered composites reached relative densities between $94-97 \%$.

The oxidation behaviour of the sintered composites was studied by thermogravimetry (TG) and differential scanning calorimetry (DSC). The TG/DSC studies were carried out using a PL Thermal Sciences system (STA-625) by raising the temperature at a rate of $10^{\circ} \mathrm{C} \mathrm{min}-1$ in flowing air $\left(40 \mathrm{~cm}^{3} \mathrm{~min}^{-1}\right)$. The phase compositions were examined by $\mathrm{X}$-ray diffraction (Philips PW 1370) (XRD) using $\mathrm{Cu} \mathrm{K \alpha}$ radiation.

The microstructure was observed by field emission type scanning electron microscopy (FE-SEM, Hitachi, S-800). The averaged distance from either Pd or YSZ to the nearest three-phase-boundary lines was determined. The length of three-phase (YSZ/Pd/ gas), $L_{\mathrm{A}}$, the contact area between $\mathrm{YSZ}$ and $\mathrm{Pd}$ phase in the bulk, $S_{\mathrm{V}}$, were determined from SEM pictures [8].

The electrical conductivity of the ZYPd40 composite was measured using a dc 4-point-method (Keithley 197 DMM). The electrical properties of the pure YSZ ceramic and ZYPd 30 were investigated using complex impedance method (Solartron 1255 frequency response analyzer). Impedance spectra were 
analyzed using the nonlinear square impedance analysis package EQUIVALENT CIRCUIT [9]. The oxygen ion transference number was measiured using an oxygen concentration cell (Nernst method) [10].

Isothermal oxygen semipermeability measurements were performed on disk-shaped samples with a diameter of $12 \mathrm{~mm}$ and thickness $2.0 \mathrm{~mm} \mathrm{[5].} \mathrm{The}$ specimens were sealed into a quartz reactor using glass rings. The data were collected in the temperature range of 900 to $1100^{\circ} \mathrm{C}$. An oxygen/nitrogen gas mixture was led over one side of the specimen, while the other side being flushed with high purity helium gas (UCAR Specialty Gases N.V., $P_{\mathrm{O}_{2}}=1.1 \times 10^{-4} \mathrm{~atm}$ ) at a precisely controlled flow rate. On-line gas chromatography (GC) (Varian model 3400) was used to analyze the oxygen and nitrogen concentrations in the effluent at the gas outlet of the permeate side. No nitrogen was detected by GC in the effluent at the permeate side, indicating that the seal was appropriate and the membrane was gas-tight. The oxygen flux was normalized for the surface area of the membrane at the feed side.

\section{Results and discussion}

\subsection{Sintering behaviour, microstructure and oxidation resistance}

The composites started to sinter at a temperature of about $1000^{\circ} \mathrm{C}$. The densification rate reached a maximum at about $1200^{\circ} \mathrm{C}$. The composites reached a density above $94 \%$ after non-isothermal sintering, the target temperature being in the range of 1350 to $1480^{\circ} \mathrm{C}$. With increasing target temperature, the density slightly increased.

The microstructure of the sintered compacts is shown in Fig. 1a-c. The results of the quantitative analysis of the microstructure are listed in Table 1. Generally speaking, shorter distances to the three phase lines and larger lengths of three phase lines are beneficial to the surface reaction. The relation between those parameters and the surface reaction rate is presently under investigation.

It was found that a slight oxidation of the palladium in the sintered dense composites only took place at the surfaces of the composites at temperatures below $877^{\circ} \mathrm{C}$. All the palladium oxide decomposed to palladium at temperatures above $877^{\circ} \mathrm{C}$. The com-
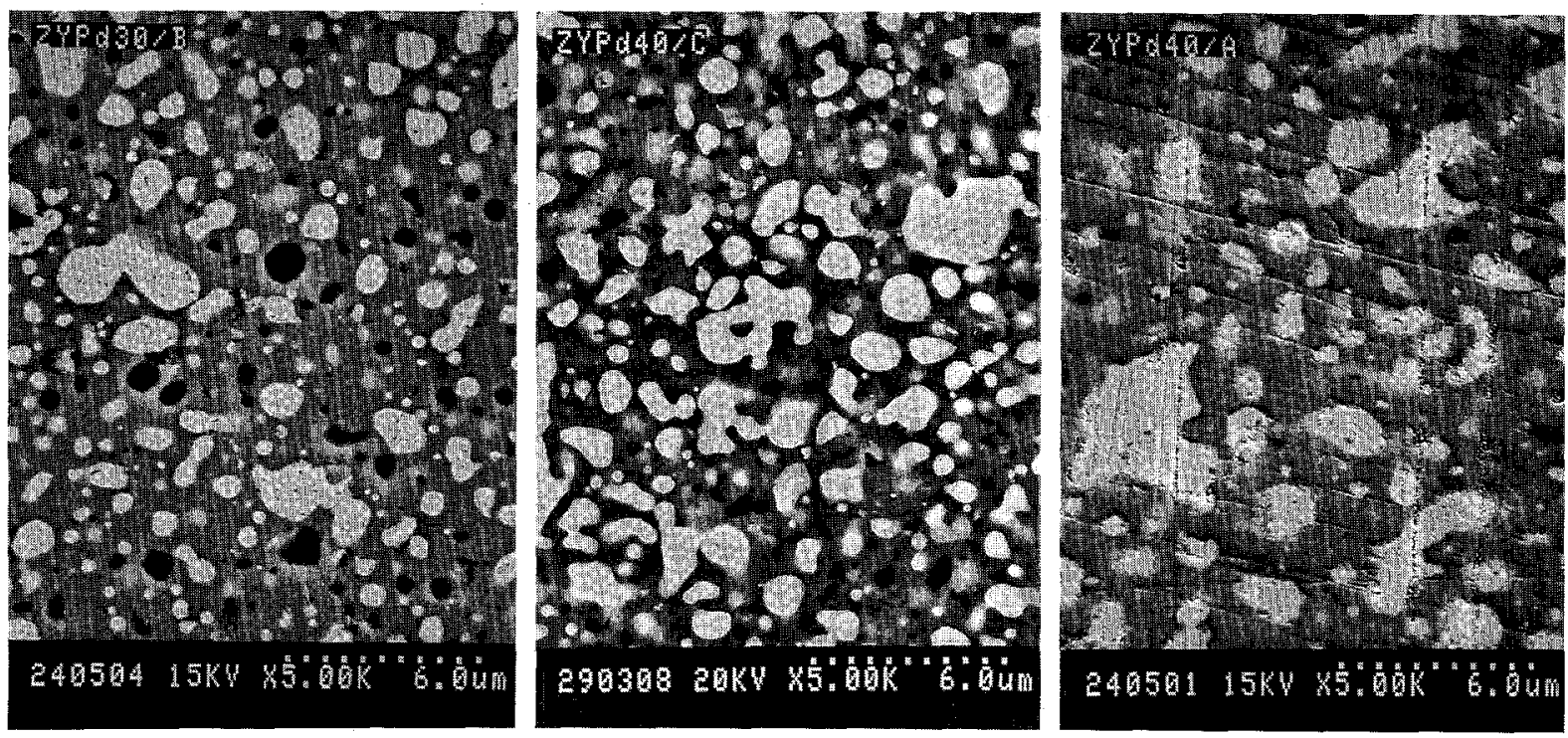

Fig. 1. Microstructure of the YSZ-Pd composites: (a) ZYPd30 sintered at $1480^{\circ} \mathrm{C}$; (b) ZYPd40 sintered at $1350^{\circ} \mathrm{C}$; (c) ZYPd40 sintered at $1480^{\circ} \mathrm{C}$. 
Table 1

Quantitative description of the microstructure of YSZ/Pd composites

\begin{tabular}{llllll}
\hline $\begin{array}{l}\text { Sample } \\
\text { No. }\end{array}$ & Preparation & $\begin{array}{l}\text { Pd content } \\
(\mathrm{vol} \%)\end{array}$ & $\begin{array}{l}L_{\mathrm{YSZ} / \mathrm{TPL}}{ }^{\mathrm{a}} \\
(\mu \mathrm{m})\end{array}$ & $\begin{array}{l}L_{\mathrm{Pd} / \mathrm{TPL}}{ }^{\mathrm{b}} \\
(\mu \mathrm{m})\end{array}$ & $\begin{array}{l}L_{\mathrm{A}}{ }^{\mathrm{c}} \\
\left(\mathrm{m} / \mathrm{m}^{2}\right)\end{array}$ \\
\hline ZYPd30B & $1480^{\circ} \mathrm{C} / \mathrm{N}_{2}$ & 30 & 0.36 & 0.26 & $\begin{array}{l}S_{\mathrm{V}}{ }^{\mathrm{a}} \\
\left(\mathrm{m}^{2} / \mathrm{m}^{3}\right)\end{array}$ \\
ZYPd40C & $1350^{\circ} \mathrm{C} / \mathrm{N}_{2}$ & 40 & 0.38 & 0.32 & $9.2 \times 10^{5}$ \\
ZYPd40A & $1480^{\circ} \mathrm{C} / \mathrm{N}_{2}$ & 40 & 0.50 & 0.44 & $1.5 \times 10^{6}$ \\
\hline
\end{tabular}

${ }^{a} L_{Y S Z / T P L}$ : diffusion length from YSZ phase to three phase lines. ${ }^{\mathrm{b}} L_{\mathrm{Pd} / \mathrm{TPL}}$ : diffusion length from Pd phase to three phase lines.

' $L_{\mathrm{A}}$ : the length of three phase lines. ${ }^{\mathrm{d}} S_{\mathrm{V}}$ : the contact area between YSZ and Pd grains in the bulk.

posites remained intact and gas-tight after being measured at elevated temperatures and experiencing rapid change in temperature for more than ten times. This demonstrated that the composites had good chemical stability and excellent thermal-shock resistance.

\subsection{Electrical conductivity of the composites}

Fig. 2 shows the total conductivities of the composites as a function of temperature. For comparison, the conductivity of YSZ is also presented.

\subsubsection{Percolative composites ZYPd40}

The conductivities of the composites were much higher than that of YSZ ceramic. For example, the conductivity of the composites at $900^{\circ} \mathrm{C}$ were about three orders of magnitude larger than that of the YSZ. This revealed that the palladium in the matrix of the

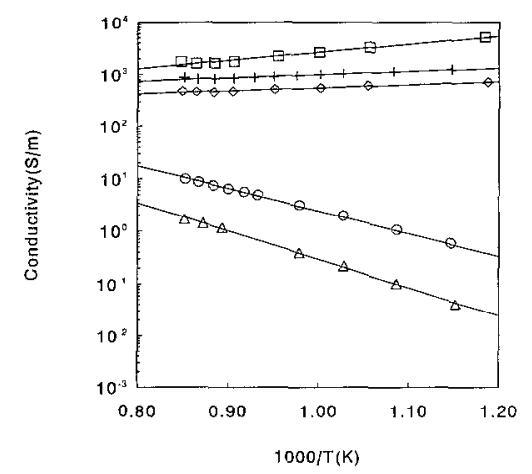

Fig. 2. The electrical conductivities of the composites and YSZ (a) ( $\square$ ) $\mathrm{ZYPd}$ sintered at $1480^{\circ} \mathrm{C}$; (b) (+) ZYPd40 sintered at $1420^{\circ} \mathrm{C}$; (c) $(\diamond) \mathrm{ZYPd} 40$ sintered at $1350^{\circ} \mathrm{C}$; (d) (O) YSZ; (e) $(\triangle) \mathrm{ZYPd} 30$ sintered at $1480^{\circ} \mathrm{C}$.
YSZ formed a percolative network, which agreed with the SEM observations (see also Fig. 1b,c).

It was also found that the total conductivity of the composites increased with increasing sintering temperature, which can be ascribed to the improvement of contact between palladium particles with increasing sintering temperature as follows from Fig. 1b,c.

\subsubsection{Non-percolative composites ZYPd30}

The total conductivity of the ZYPd30 composite was about one order of magnitude smaller than that of the YSZ. The transference number of oxygen ions as determined using the EMF method was close to $100 \%$. This gave solid evidence of a non-percolation network for the palladium phase, which agreed with the SEM observation (see Fig. 1a).

\subsection{Oxygen permeation of YSZ-Pd composites}

The percolative composite shows a much larger oxygen permeability than that of the non-percolative composite. For a $2.0 \mathrm{~mm}$ thick percolative sample (ZYPd40), an oxygen flux of $4.3 \times 10^{-8} \mathrm{~mol} / \mathrm{cm}^{2} / \mathrm{s}$ was measured at $1100^{\circ} \mathrm{C}$ with oxygen partial pressures at the feed and permeate sides being 0.209 and $0.014 \mathrm{~atm}$, respectively. This value is about two orders of magnitude larger than that observed for a 2.0 mm thick non-percolative composite (ZYPd30) at the same temperature with the oxygen partial pressures at the feed and permeate sides being 0.209 and $1.5 \times 10^{-4} \mathrm{~atm}$, respectively. The difference in the oxygen permeability between the percolative and nonpercolative composites can be explained as follows. In order to sustain oxygen permeation, the transport of oxygen ions must be accompanied by a simultaneous movement of electrons or electronic holes to 
maintain electrical neutrality. For percolative composites, oxygen ions transport through the YSZ phase while the electrons move fast through the percolative palladium phase to maintain electrical neutrality, resulting in a large oxygen permeation flux. However for non-percolative composites, the electrical neutrality can only be maintained by the relatively slow movement of electron holes through the YSZ phase.

\subsubsection{Temperature dependence of the oxygen permeation}

As shown in Fig. 3, the oxygen flux through the composites increases with temperature. A value of $82.6 \mathrm{~kJ} / \mathrm{mol}$ for the apparent activation energy was determined from the temperature dependence of the oxygen flux through the percolative composite (ZYPd40). This value is very close to the activation energy $(82.7 \mathrm{~kJ} / \mathrm{mol})$ for electrical conduction of oxygen ions in the YSZ ceramic calculated from Fig. 2. This indicates that the rate limiting step of oxygen permeation via ZYPd40 was the diffusion of oxygen ions through the YSZ phase. For the non-percolative composite (ZYPd30), the activation energy was about $170 \mathrm{~kJ} / \mathrm{mol}$, suggesting that the transport of electron holes through the YSZ phase was the rate limiting step of the oxygen permeation through the non-percolative system.

\subsubsection{Pressure dependence of the oxygen permeation}

As can be seen in Fig. 4, the order of the oxygen partial pressure dependence of the permeability of ZYPd40, i.e., $n$, is only about 0.06 . The very small

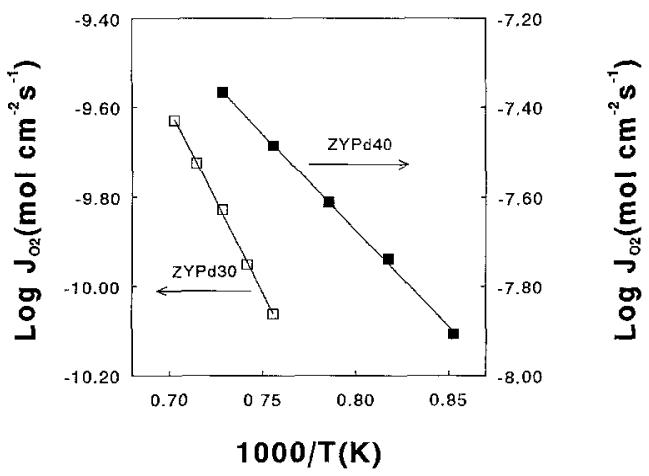

Fig. 3. Temperature dependence of the oxygen flux through YSZPd composites.

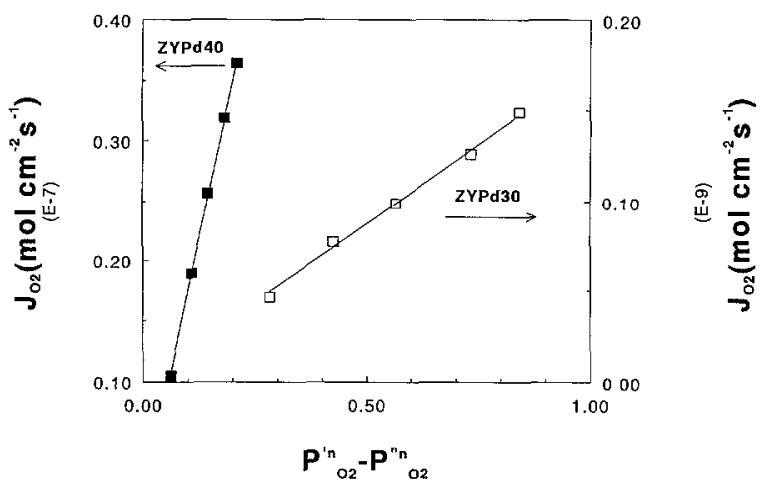

Fig. 4. Pressure dependence of the oxygen flux through YSZ-Pd composites

value of $n$ again suggests that the bulk process of the oxygen permeation via the percolative composite ZYPd40 with a thickness of $2 \mathrm{~mm}$ was the rate limiting step.

The order of oxygen partial pressure dependence of the permeability of $\mathrm{ZYPd} 30$ was about $1 / 4$. This also strongly suggest that the transport of electron holes through the YSZ phase in the non-percolative composite is the rate limiting step. It can be concluded that the mechanism of the oxygen permeation via non-percolative composite $\mathrm{ZYPd} 30$ is similar to that of lime-stabilized zirconia [11].

\section{Conclusions}

(i) Palladium phase in the matrix of YSZ containing $40 \mathrm{vol} \% \mathrm{Pd}$ was percolative, but non-percolative when its content was reduced to $30 \mathrm{vol} \%$.

(ii) The percolative composite ZYPd40 showed a high oxygen permeability, which was about two orders of magnitude larger than that of the non-percolative composite $\mathrm{ZYPd} 30$. The rate limiting step of the permeation via $Z Y P d 40$ is the transport of the oxygen ions through the YSZ phase.

(iii) It has been proved that the concept of the oxygen permeation via percolative dual phase system is workable, with oxygen ions running through the ionic conductive phase, the electrical charge being balanced by simultaneous transport of electrons through the electronically conducting phase. 


\section{Acknowledgements}

The investigations were supported by the Netherlands Foundation for Chemical Research with financial aid from the Netherlands Organization for Scientific Research (NWO). The experimental support provided by Dr. G.H.M. van de Velde (TGA/DSC), Mr. J. Boeijsma (XRD), Mr. M. Smithers (SEM), Mr. R. van Doorn (oxygen permeation) and Dr. K.J. de Vries (transference number measurement) are gratefully acknowledged.

\section{References}

[1] A.J. Burggraaf, H.J.M. Bouwmeester, B.A. Boukamp, R.J.R. Uhlhorn and V. Zaspalis, in: Science of Ceramic Interfaces, ed. J. Nowotny (Elsevier, Amsterdam, 1991) p. 525.

[2] Y.M. Gryaznov, V.J. Vedernikov, Kinet. Catal. 27 (1985) 129.
[3] T.J. Mazanec, T.L. Cable and J.G. Frye Jr., Solid State Ionics 53-56 (1992) 111.

[4] L. Heyne, in: Solid Electrolytes, Topics in Applied Physics, ed. S. Geller (Springer, Berlin, Heidelberg, New York, 1977) p. 169 .

[5] H.J.M. Bouwmeester, H. Kruidhof, A.J. Burggraaf and P.J. Gellings, Solid State Ionics 53-56 (1992) 460.

[6] M.J. Verkerk, M.W.J. Hammink and A.J. Burggraaf, J. Electrochem. Soc. 130 (1983) 70.

[7] M.J. Verkerk and A.J. Burggraaf, J. Electrochem. Soc. 130 (1983) 78 .

[8] W.D. Kingery, H.K. Bowen and D.R. Uhlmann, Introduction to Ceramics, 2nd Ed. (Wiley, New York, 1976) p. 527 .

[9] B.A. Boukamp, Solid State Ionics 18/19 (1986) 136.

[ 10 ] K.J. de Vries, T. Van Dijk and A.J. Burggraaf, in: Fast Ion Transport in Solids, eds. P Vashishta, J.N. Mundy and G.K. Shenoy (Elsevier, Amsterdam, 1979) p. 679.

[11] S. Dou, C.R. Masson and P.D. Pacey, J. Electrochem. Soc. $132(1985) 1843$. 